\title{
Adaptación y validación del cuestionario de barreras para la búsqueda de ayuda para problemas de salud mental en adolescentes chilenos* $^{*}$
}

\section{Adaptation and Validation of Barriers to Adolescents Seeking Help} Scale for Mental Health Problems in Chilean Teenagers

\author{
Cencilia Olivari ${ }^{\mathrm{a}}$ \\ Universidad Católica del Maule, Chile \\ ORCID: http://orcid.org/0000-0001-5632-3886 \\ Mónica Guzmán-GonzÁlez \\ Universidad Católica del Norte, Chile
}

a Autor de correspondencia. Correo electrónico: cecilia.olivari@yahoo.es

Para citar este artículo: Olivari, C., \& GuzmánGonzález, M. (2018). Adaptación y validación del cuestionario de barreras para la búsqueda de ayuda para problemas de salud mental en adolescentes chilenos. Universitas Psychologica, 17(1), 1-12. https:// doi.org/10.11144/Javeriana.upsy17-1.avcb

\section{RESUMEN}

La presente investigación tuvo como objetivo, adaptar y validar en adolescentes chilenos, el cuestionario de barreras para la búsqueda de ayuda para problemas de salud mental, versión breve (BASH-B). Este cuestionario mide las barreras o razones percibidas por los adolescentes para no buscar ayuda profesional cuando presentan algún problema psicológico. Los participantes fueron 387 adolescentes de entre 14 y 19 años $(M=15.71$ años), escolarizados en establecimientos de enseñanza media de la comuna de Talca (Chile). Se analizaron las propiedades psicométricas del instrumento. El análisis confirmatorio corroboró la estructura factorial unidimensional. Además, los índices de confiabilidad fueron adecuados. La escala presentó asociación significativa con las variables depresión y autoeficacia en las direcciones esperadas. En base a estos resultados, es posible decir que el BASH-B es un instrumento que posee propiedades psicométricas adecuadas para medir las barreras de la búsqueda de ayuda en el ámbito de la salud mental en adolescentes.

Palabras clave

búsqueda de ayuda; Barreras; Salud Mental; Adolescentes; Validación de instrumento.

\begin{abstract}
This research has a goal to adapt and validate for Chilean teenagers the Barriers to Adolescents Seeking Help Scale, brief version (BASH-B). This questionnaire measures the perceived barriers and reasons for teenagers not looking for professional help when they presents some psychological problem. There were 387 teenagers participants between 14 and 19 years olds $(M=15.71$ years $)$, attending high schools in Talca regional area. The psychometric properties of the instrument were analyzed. A confirmatory factor analysis corroborated the one-dimensional structure. The reliability indexes as appropriate. In addition, the scale presented significant association with the variables depression and self-efficacy in the hipothesized directions. Based on these results is possible to confirm
\end{abstract}


that the BASH-B a appropriate psychometric properties to measure the barriers to help seeking for Mental health Problems in teenagers.

Keywords

help-Seeking; Barriers; Mental Health; Adolescent; Test Adaptation.

La búsqueda de cuidados y ayuda profesional en salud es una conducta que será determinante para promover el bienestar de las personas (Baker, 2007; OMS, 2010). La conducta de búsqueda de ayuda oportuna en los jóvenes es fundamental para su salud mental (Abram, Paskar, Washburn, \& Teplin, 2008; Barker, Olukoya, \& Aggleton, 2005). No obstante, un gran número de adolescentes y jóvenes no busca ayuda profesional, a pesar de tener la necesidad de recibir ayuda (Abram et al., 2008; Carlisle, Shickle, Cork, \& McDonagh, 2006; Lindberg, Lewis-Spruill, \& Crownover, 2006; Lyren, Kodish, Lazebnik, \& O'Riordan, 2006). Un ejemplo de esto, aporta el estudio de Gulliver, Griffiths, y Christensen (2010), quienes realizan una revisión sistemática de las barreras para la búsqueda de ayuda en salud mental en adolescentes y jóvenes, que evidencia que solo entre un 18 a $34 \%$ de jóvenes que presentan altos niveles de síntomas depresivos o de ansiedad, buscan ayuda profesional. Este rechazo constituye uno de los mayores obstáculos para la prevención de temas como el suicidio adolescente o el desarrollo de distintas psicopatologías (Baker, 2007). Además, el no buscar ayuda profesional de manera oportuna está asociado a la evolución de una larga historia del problema de salud mental, y con presentar un mayor número de recaídas cuando se inicia un tratamiento (Hunt \& Eisenberg, 2010). Esta baja utilización de ayuda profesional lleva al interés de estudiar los motivos o barreras por las cuales los adolescentes, a pesar de presentar un problema en salud mental, no buscan ayuda.

\section{Barreras para la búsqueda de ayuda}

Se definen las "barreras" como aquellas variables que inhiben tanto la utilización de los servicios de ayuda o consejería, como el obtener resultados exitosos de estos servicios (Abram et al., 2008)

A nivel internacional, existe un buen nivel de desarrollo de investigación, que ha logrado identificar distintas barreras para la búsqueda de ayuda, entre las que se tienen: a) Factores Estructurales: incluye factores financieros, la disponibilidad de servicios de salud mental y disponibilidad de tiempo (Rickwood, Deane, Wilson, \& Ciarrochi, 2005), b) Experiencia Previa de ayuda profesional en salud mental: se ha evidenciado que el tener una experiencia pasada negativa (poco satisfactoria) es una barrera para la búsqueda de ayuda profesional (Lindsey et al., 2006; Raviv, Raviv, VagoGefen, \& Fink, 2009; Rickwood et al., 2005), c) Necesidad de autonomía: se refiere a las creencias que tiene el adolescente de ser autónomo y tener control personal (Vogel, Wester, \& Larson, 2007). Las investigaciones evidencian que los adolescentes prefieren confiar y depender de sí mismos más que buscar ayuda externa para sus problemas, y que no desean que sus padres conozcan los mismos (Gulliver et al., 2010; Wilson \& Deane, 2012), d) Alfabetización en temas de salud mental: se refiere al grado de conocimiento y creencias sobre los problemas de salud mental que ayudan a su reconocimiento, manejo y prevención (Wilson, Rickwood, Bushnell, Caputi, \& Thomas, 2011). Se ha evidenciado, en diversos estudios, que el tener una alfabetización incompleta de salud mental constituye una importante barrera para la búsqueda de ayuda y el acceso a servicios profesionales (Burns \& Rapee, 2006; Ranahan, 2010; Wilson et al., 2011; Wilson, Deane, Marshall, \& Dalley, 2008), e) Síntomas de Malestar emocional y/o trastornos emocionales: pueden actuar como una barrera para la búsqueda de ayuda (Ciarrochi, Deane, Wilson, \& Rickwood, 2002; Wilson \& Deane, 2010). Así, se ha reportado que la búsqueda de ayuda estaría inversamente correlacionada con el grado de distress o malestar psicológico percibido por 
los jóvenes (Ciarrochi et al., 2002; Tishby, Turel, Gumpel, \& Pinus, 2001). Lo mismo ocurre, respecto a la presencia de síntomas depresivos como una forma de malestar emocional; algunos estudios han logrado identificar que, mayores niveles de síntomas depresivos y desesperanza, se asocian negativamente con la intención de buscar ayuda (Wilson \& Deane, 2010; Wilson, Rickwood, \& Deane, 2007), f) Estigma: las creencias negativas y prejuicios hacia los problemas de salud mental han sido identificados como una importante barrera para buscar ayuda (Abram et al., 2008; Boyd et al., 2007; Eisenberg, Downs, Golberstein, \& Zivin, 2009), g) Confidencialidad: el temor o preocupación de que el profesional de la salud pueda romper la confidencialidad es una barrera frecuentemente mencionada por los adolescentes para no buscar ayuda (Gulliver et al., 2010; Lyren et al., 2006)

Respecto al estudio de las barreras para la búsqueda de ayuda para problemas de salud mental en población específica adolescente, prácticamente no existen medidas que permitan evaluarlas.

\section{Cuestionario de Barreras para la Búsqueda de ayuda}

Un instrumento específico para evaluar las barreras percibidas por los adolescentes para buscar ayuda en temas de salud mental, es el Cuestionario de Barreras para la búsqueda de ayuda, versión breve (BASH-B) (Wilson, Deane, Ciarrochi, \& Rickwood, 2005). El BASH-B se desarrolló a partir del cuestionario para búsqueda de ayuda de Kuhl, Jarkon-Horlick, y Morrissey (1997), que estaba compuesto por 37 ítems, que fueron planteados a partir de la revisión de la literatura respecto a las barreras que, a través de distintas investigaciones, se habían identificado como obstáculos para la búsqueda de ayuda. No obstante, al utilizar el instrumento, obtuvieron un alto alfa de Cronbach (0.91), que les hizo advertir la redundancia de ítems, y llevaron a estos autores a desarrollar una versión breve para evitar la superposición de estos. La versión breve, que tendría una estructura unidimensional, quedó compuesta por 11 ítems, los que fueron seleccionados a partir de una aplicación piloto que hizo identificar las barreras que más frecuentemente señalaban los adolescentes (autonomía, vergüenza y estigma, limitaciones económicas y de tiempo, temor a la ruptura de la confidencialidad, temor a que la familia tome conocimiento, la creencia que nada pueda ayudarlo(a), y coerción por parte del profesional de la salud). Posteriormente, Wilson y Deane (2012) identificaron una estructura factorial de 2 escalas: escala de "necesidad de autonomía”, que incluyó 2 ítems, y una escala de "temores (o aprensiones) hacia la búsqueda de ayuda". Este instrumento ha sido utilizado en diversos estudios sobre barreras para la búsqueda de ayuda en salud mental, tanto en su versión original (Leshem, Haj-Yahia, \& Guterman, 2015; Raviv et al., 2009), como la breve (Cartmill, Deane, \& Wilson, 2009; Wilson \& Deane, 2012; Wilson et al., 2011). En habla hispana, no existe ningún instrumento que posibilite medir estas barreras y poder progresar en el tema de búsqueda de ayuda en este grupo, por esto se considera relevante el realizar la adaptación y validación del BASH-B.

En base a lo anterior, el objetivo del presente estudio fue adaptar transculturalmente la versión en inglés del BASH-B, y luego estudiar sus propiedades psicométricas para poder contar con un instrumento que sea válido y confiable en nuestro medio, y así poder seguir estudiando las barreras para la búsqueda de ayuda para temas o problemas de salud mental en ese grupo etario.

\section{Método}

\section{Participantes}

Se efectuó una investigación no experimental, transversal y correlacional. La muestra fue no probabilística, y estuvo constituida por 387 adolescentes, 247 mujeres y 138 hombres (2 personas omitieron la información) de 14 a 19 años $(M=15.71, D E=1.4)$, que estaban cursando de primero a cuarto de enseñanza media de 4 distintos establecimientos 
educacionales de enseñanza media diurna de la ciudad de Talca. Un $19.4 \%(N=72)$ provenía de Liceo Técnico, y un $80.6 \%$ del Liceo Científico Humanista de colegios municipales y particulares subvencionados.

\section{Procedimiento}

En una primera etapa, se procedió a la adaptación transcultural del cuestionario, para lo cual se siguieron los siguientes pasos: a) establecimiento de contacto con el autor original del cuestionario, quien dio la autorización para llevar a cabo la adaptación y validación del cuestionario, b) Traducción del texto original (del inglés al español) por 2 personas bilingües del área de la psicología y salud mental, y que trabajaron de manera independiente, c) Retro traducción (de la primera versión en español al inglés) por 2 traductores nativos, d) Sometimiento a jueces expertos para evaluar nivel de comprensión de ítems, redacción de estos y grado de ajuste de ítems a cada factor de la escala.

Luego de obtener esta versión revisada por jueces expertos, se aplicó por medio de entrevistas cognitivas a una muestra de 20 adolescentes, y luego se aplicó de manera grupal a una muestra piloto de 50 estudiantes de enseñanza media, con el fin de advertir posibles problemas de comprensión de los ítems.

En una segunda etapa, se envió una carta de invitación, y se solicitó entrevista a 9 establecimientos educacionales de la comuna de Talca, de los cuales 4 autorizaron que se invitara a los alumnos a participar. Una vez obtenida la autorización, se convocó a participar, y se solicitaron los respectivos consentimientos y asentimientos. Los cuestionarios fueron aplicados en la sala de clases de manera colectiva por 2 psicólogos y 2 alumnos de último año de la carrera de psicología, previamente entrenados en la aplicación de estos. El tiempo de aplicación de los cuestionarios fue de 30 a 40 minutos aproximadamente. Esta investigación fue aprobada por el comité de ética de la Universidad Católica del Maule.

\section{Instrumentos}

Además de un cuestionario sociodemográfico, en que se incluyó la edad, género y establecimiento educacional, se aplicaron los siguientes instrumentos:

Cuestionario de Barreras para la búsqueda de ayuda, versión Breve (BASH-B)

Esta es una escala de auto reporte, desarrollada por Wilson et al. (2005). Contiene 11 ítems que describen barreras percibidas para la búsqueda de ayuda profesional, y deriva de la escala del mismo nombre desarrollada por Kuhl et al. (1997). Cada ítem es evaluado en una escala Likert de 6 puntos $(1=$ altamente en desacuerdo a $6=$ altamente de acuerdo). Un mayor puntaje indica percepción de más barreras para la búsqueda de ayuda. En su estudio de validación, el puntaje obtenido a través del alfa de Cronbach reportado fue de 0.83 . Respecto a su validez, se han encontrado correlaciones inversas con experiencias insatisfactorias previas de psicoterapia (Wilson et al., 2005), y correlaciones directas con sintomatología depresiva (Wilson et al., 2005; Wilson \& Deane, 2012).

\section{Escala de Autoeficacia General}

Busca medir un sentido general y estable de autoeficacia, es decir, la creencia de que uno puede realizar tareas nuevas o difíciles o superar la adversidad en diversas áreas. Es un instrumento de autoreporte de 10 ítems, que son contestados mediante una escala Likert de 4 puntos, de acuerdo a lo que esta percibe de su capacidad en ese momento. Las respuestas van desde Incorrecto (1 punto) a Cierto (4 puntos). El puntaje mínimo es de 10 puntos, y el máximo es de 40 puntos. En esta escala, a mayor puntaje, mayor autoeficacia general. Esta escala ha sido utilizada en diversas culturas, en población adulta y adolescente desde los 12 años de edad. Respecto a su validez, se han encontrado relaciones positivas con emociones favorables, 
autorregulación, y autoestima, entre otros (Cid, Orellana, \& Barriga, 2010; Luszczynska, Scholz, $\&$ Schwarzer, 2005). Respecto a la confiabilidad por consistencia interna, se han obtenido en muestras de 23 naciones coeficientes, alfas de Cronbach que van desde 0.76 a 0.9. (Luszczynska et al., 2005). En su adaptación para población española, (Sanjuán Suárez, PerezGarcía, \& Bermúdez Moreno, 2000) obtuvieron un coeficiente alfa de 0.87 , mientras que en su estudio de validación en Chile, Cid et al. (2010) obtuvieron un coeficiente alfa de 0.84 .

\section{Cuestionario de Sintomatología Depresiva}

Se aplicó la versión chilena de la Escala CES$\mathrm{D}$, del centro de estudios epidemiológicos de Estados Unidos, validada en chile por Gempp, Avendaño, y Muñoz (2004). Este instrumento mide frecuencia de sintomatología depresiva y ánimo depresivo durante las últimas 2 semanas. Es una escala de 20 ítems, con 4 alternativas de respuesta en formato Likert. Esta escala está validada en población juvenil chilena. El índice de confiabilidad de la escala completa fue de un coeficiente de consistencia interna alfa de Cronbach de 0.87. La escala ha demostrado capacidad para discriminar entre sujetos normales y depresivos (Gempp et al., 2004).

\section{Resultados}

\section{Plan de Análisis}

De manera previa a la ejecución de los análisis principales, se realizó un examen preliminar de los datos. Del total de 404 participantes en el estudio, 17 fueron removidos por poseer datos ausentes. Por otra parte, se buscó identificar atípicos multivariados mediante el estadístico de Mahalanobis, sin embargo, ningún caso alcanzó el valor crítico $(<0.001)$. De esta manera, el análisis se realizó sobre un total de 387 participantes.
Posteriormente, se procedió a describir la muestra en relación con las variables evaluadas, y a examinar diferencias por sexo.

Para la selección del número de factores a retener, se ocuparon dos estrategias. En primer lugar, se llevó a cabo un Análisis Paralelo (Horn, 1965), un procedimiento más exacto para determinar el número de factores del criterio de Kaiser, ocupando el programa Factor 8.02 (Lorenzo-Seva \& Fernando, 2006).

Posteriormente, se testearon y compararon mediante modelos anidados, a través de un análisis factorial confirmatorio, dos soluciones alternativas; la primera, con una estructura unidimensional, tal como la propone el autor original del instrumento en otros estudios (Cartmill et al., 2009; Lubman et al., 2016), y luego, una de dos factores, que fue identificada en un estudio posterior (Wilson \& Deane, 2012).

Como indicadores de ajuste, se consideraron el RMSEA y su intervalo de confianza del 90 $\%$, el Comparative Fit Index (CFI), el Root Mean Square Error of Approximation (RMSEA) y el Standardized Root Mean Square Residual (SRMR). Se definieron como criterios de buen ajuste valores sobre 0.95 en el CFI, y cercanos o menores a 0.60 en el caso del RMSEA y el SRMR, de acuerdo a lo propuesto por (Bentler, 1990).

La diferencia en el valor de chi cuadrado fue ocupada como dato estadístico para calcular la diferencia entre modelos anidados.

Una vez que se determinó el modelo más adecuado y parsimonioso, se estimó el índice de fiabilidad de la escala y se calcularon los coeficientes de correlación de la escala con variables teóricamente asociadas, en este caso, depresión y autoeficacia.

\section{Estadísticos Descriptivos y Diferencias según Sexo}

En la Tabla 1, se presenta la estadística descriptiva de las variables evaluadas. 


\section{Tabla 1}

Estadística descriptiva de las escalas y diferencias entre hombres y mujeres

\begin{tabular}{ccccccccc}
\hline \multicolumn{1}{c}{ Variable } & M & DE & Min & Max & Asimetría & Curtosis & $t$ & IC 95\% \\
\hline BASH total & 36.27 & 10.26 & 11 & 64 & 0.01 & -0.22 & 1.6 & $-0.399-3.88$ \\
Depresión & 21.38 & 12.19 & 0 & 56 & 0.47 & -0.63 & $4.95^{*}$ & $3.75-8.69$ \\
Autoeficacia & 29.46 & 5.61 & 11 & 40 & -0.39 & -0.21 & $-2.02^{*}$ & $-2.35--0.29$ \\
\hline \multicolumn{8}{c}{$N=387 ; * p<0.05$}
\end{tabular}

Además, se evaluó si existían diferencias entre hombres y mujeres en las variables en estudio, mediante la prueba $t$ de diferencias de medias para muestras independientes.

De acuerdo a este análisis, no se detectaron diferencias en la escala BASH-B en función del sexo, $t_{(383)}=1.6, p=0.11$.

Sin embargo, respecto a los niveles de depresión, se detectaron diferencias estadísticamente significativas, $t$ (396) $=4.95$, $p<0.001$, d de Cohen $=0.54$, siendo las mujeres quienes reportaron puntajes más altos en esta variable, $(M=23.6, D E=12.52)$ que los hombres, $(M=17.37, D E=10.47)$. Por otra parte, también se identificaron diferencias estadísticamente significativas en cuanto a la autoeficacia, $t_{(396)}=-2.45, p=0.015, \mathrm{~d}$ de Cohen $=0.22$, con los hombres reportando mayores niveles de autoeficacia $(M=30.28, D E$ $=5.34)$ que las mujeres, $(M=29.09, D E=$ 5.66).

No se detectaron diferencias en el BASH$\mathrm{B}, \mathrm{F}_{(2,384)}=1.66, p=0.19$, según nivel socioeconómico, ni tampoco asociación entre la edad y las barreras en la búsqueda de ayuda $(p>$ 0.05).

\section{Validez de constructo}

Para examinar la estructura factorial del BASH, primero realizó un análisis paralelo (Horn, 1965), con el fin de identificar la cantidad de factores que se deberían retener.

Los resultados del análisis paralelo, que ocuparon una matriz de correlación de 500 muestras aleatorias, indican que el autovalor empírico del primer factor real (3.29), se encuentra por encima del promedio (1.28) y del percentil 95 de los autovalores aleatorios (1.35). A partir del segundo factor, se identifica que el segundo factor real (1.26) se encuentra por encima del promedio (1.2), pero por debajo del percentil 95 (1.25), de los segundos autovalores aleatorios. Al considerar estos resultados, y ocupar el criterio más robusto, la solución más adecuada sería de un único factor; sin embargo, dado que la comparación de las medias de los autovalores aleatorios y reales propone dos dimensiones, se decidió estudiar la diferencia en el ajuste entre los modelos de uno y dos factores, con base en antecedentes psicométricos previos, que han identificado estructuras uni (Wilson et al., 2005) y bidimensionales para la escala en cuestión (Wilson \& Deane, 2012).

El resultado de este análisis fue complementado con un análisis de las diferencias en el ajuste entre modelos anidados, ocupando análisis factorial confirmatorio. El análisis se realizó sobre el total de los ítems, ocupando datos completos y con Máxima Verosimilitud como método de estimación.

El ajuste del modelo de un factor (Modelo 1), evidenció un pobre ajuste a los datos, $\chi 2(44, \mathrm{~N}$ $=387)=169.771, p<0.001 ; \mathrm{CFI}=0.819$; RMSEA $=0.086,90 \%$ IC $[0.073,0.01] ;$ SRMR $=0.059$.

La revisión de los índices de modificación sugiere la existencia de dos errores correlacionados de magnitud considerable, entre los ítems 1 ("Si tuviera un problema, lo resolvería yo solo/a") y 11 ("Creo que yo mismo/a debería resolver mis propios problemas"), y entre los ítems 3 ("Si recibiera ayuda profesional por un problema psicológico, me preocupa que el profesional no mantenga mi problema en secreto") y 4 ("Si recibiera ayuda profesional, me preocupa que tal vez tuviera que hacer o decir algo que yo no quería") (rango de los Índices de Modificación $=$ 50.016-50.018).

Se estimó luego un segundo modelo (Modelo 2), con dos dimensiones (necesidad de autonomía y temores hacia la búsqueda de ayuda), de acuerdo con lo identificado por Wilson y Deane (2012), el donde se evidenció también un mal ajuste a los datos, $\chi 2(43, N$ $=387)=118.741, p<0.001 ; \mathrm{CFI}=0.891$; RMSEA $=0.067,90 \%$ IC $[0.053,0.082]$; SRMR $=0.049$. En este modelo, los índices 
de modificación nuevamente dan cuenta de la existencia de un error correlacionado entre los ítems 3 y $4(\mathrm{IM}=50.48)$.

Considerando estos resultados, se optó por examinar un tercer modelo, unidimensional, en el que se permitiese la correlación entre los pares de errores sugeridos por los índices de modificación, dado que la revisión de ambos da cuenta de un traslape en sus contenidos. Tal decisión se fundamentó en que las covarianzas de error pueden especificarse cuando ellas son susceptibles de ser explicadas sustantivamente (Brown, 2016), y en donde además, un factor debe estar compuesto por al menos tres ítems.

Este tercer modelo (Modelo 3), que incluyó la correlación entre los errores de ambos pares de ítems, tuvo un ajuste fue adecuado, $\chi 2$ (42, $N=387)=66.944, p<0.001 ; \mathrm{CFI}=0.964$; RMSEA $=0.039,90 \%$ IC $[0.02,0.056]$; SRMR $=0.036$. Las cargas factoriales de esta nueva solución fueron significativas $(p<0.05)$, y los índices de modificación no identifican otras áreas problemáticas.

De esta manera y al observar la Tabla 2, donde se presentan los modelos anidados, se observa que el modelo 3 es el que presenta un ajuste significativamente mejor.

Considerando estos resultados, y con base en antecedentes psicométricos y teóricos previos acerca de la unidimensionaldad de la escala (Wilson et al., 2005), se optó por una solución unifactorial (Modelo 3) como la más aceptable y de mayor parsimonia. Los resultados de este análisis se observan en la Tabla 3.

Tabla 2

Índices de Ajuste para Modelos anidados con AFC

\begin{tabular}{rcccccccc}
\hline Modelo & $\chi^{2}$ & gl & $p$ & $\Delta \chi^{2}$ & $\Delta$ gl & $\Delta_{\text {valor }-p}$ & CFI & RMSEA / IC 90 \% \\
\hline Modelo 1 & 169.77 & 44 & 0 & - & - & & 0.819 & $0.09[0.07-0.1]$ \\
Modelo 2 & 118.74 & 43 & 0 & 51.03 & 1 & 0 & 0.891 & $0.07[0.05-0.08]$ \\
Modelo 3 & 66.94 & 42 & 0 & 51.08 & 1 & 0 & 0.964 & $0.04[0.02-0.06]$ \\
\hline
\end{tabular}

Nota. Modelo 1: Un factor; Modelo

2: Dos factores; Modelo 3: Un factor con dos errores correlacionados
Tabla 3

Cargas factoriales estandarizadas de los ítems del BASH mediante un Análisis Factorial Confirmatorio

\begin{tabular}{lc}
\hline \multicolumn{1}{c}{ Ítem } & $\begin{array}{c}\text { Carga } \\
\text { Factorial }\end{array}$ \\
\hline $\begin{array}{l}\text { 1. Si tuviera un problema, lo resolvería solo/a } \\
\text { 2. Aunque quisiera recibir ayuda profesional, yo } \\
\text { no tendría el tiempo para asistir a las sesiones y } \\
\text { recibir esta ayuda }\end{array}$ & 0.33 \\
3. Si recibiera ayuda profesional por un problema \\
psicológico, me preocupa que el profesional no \\
mantenga mi problema en secreto \\
$\begin{array}{l}\text { 4. Si recibiera ayuda profesional, me preocupa } \\
\text { que tal vez tuviera que hacer o decir algo que yo } \\
\text { no quería }\end{array}$ \\
$\begin{array}{l}\text { 5. Si estuviera recibiendo ayuda profesional, no } \\
\text { querría que mi familia lo supiera nunca }\end{array}$ \\
$\begin{array}{l}\text { 6. Los adultos realmente no pueden entender los } \\
\text { problemas que tenemos los jóvenes }\end{array}$ & 0.44 \\
7. Aunque tuviera un problema psicológico, me \\
sentiría avergonzado/a de buscar ayuda \\
profesional
\end{tabular}

\section{Confiabilidad}

La fiabilidad de la escala, mediante el alpha de Cronbach, fue de 0.76, y con un coeficiente Omega de McDonald de 0.77, 95 \% IC [0.723, 0.799], estimado mediante el programa Mplus.

\section{Validez de criterio}

La validez de criterio fue examinada analizando la asociación del BASH, con las variables depresión y autoeficacia. Tal como se observa en la Tabla 4, los puntajes en el BASH se asociaron inversamente con la autoeficacia $(r=-0.16$, $p<0.01$ ), y directamente con los niveles de depresión $(r=0.42, p<0.01)$. 
Tabla 4

Correlaciones entre las subescalas del BASH y los niveles de depresión y autoeficacia

\begin{tabular}{lcc}
\hline \multicolumn{1}{c}{ Variable } & 1 & 2 \\
\hline 1. BASH total & - & \\
2. Depresión & $0.42 * *$ & - \\
3. Autoeficacia & $-0.16^{*}$ & $-0.32 * *$ \\
\hline
\end{tabular}

$\mathrm{N}=387 ;{ }^{*} p<0.05: * p<0.01$

\section{Discusión}

El propósito de este estudio fue reportar el proceso de adaptación y validación al castellano, de las puntuaciones del cuestionario de Barreras para la búsqueda de ayuda para temas de salud mental, versión breve del BASH-B. Para esto, primero se realizó un proceso de adaptación transcultural del cuestionario, para luego ser aplicado a una muestra de adolescentes escolarizados de la ciudad de Talca (Chile).

El análisis confirmatorio realizado, corrobora la estructura unidemensional original del cuestionario. Si bien se probó la estructura de 2 factores, identificada en el estudio de Wilson y Deane (2012), en el presente estudio tuvo un mejor ajuste el modelo de un factor. Por consiguiente, la escala chilena queda constituida por los mismos ítems que la escala en su versión original en inglés.

Respecto a la validez de criterio, se obtuvieron, además, correlaciones significativas tanto con sintomatología depresiva como con autoeficacia. Como era de esperarse, la sintomatología depresiva se correlacionó de manera directa con las barreras de búsqueda de ayuda. Es decir, los adolescentes que puntuaron más alto en sintomatología depresiva son los que más barreras perciben para la búsqueda de ayuda. Esto coincide con distintas investigaciones que han señalado que, a mayor sintomatología emocional, los adolescentes perciben más barreras para buscar ayuda y, por tanto, solicitan menos ayuda tanto informal como formal (Wilson et al., 2007) A esta situación de tener necesidad de buscar ayuda para un problema emocional y no hacerlo, se le ha denominado "el proceso de negación de ayuda" (Carlton \& Deane, 2000; Wilson et al., 2005).

Por el contario, y como era de esperarse, se obtuvo una relación inversa del puntaje total del BASH-B con autoeficacia, lo que indica que los adolescentes que presentan un mayor nivel de autoeficacia, perciben menos barreras para buscar ayuda.

Finalmente, si bien no era un objetivo en el estudio, se examinaron posibles diferencias de género en las variables incluidas en el estudio. No se observaron diferencias significativas en cuanto a barreras para la búsqueda de ayuda, como fue reportado en el estudio de Wilson y Deane (2012). Sin embargo, se advirtieron diferencias significativas en autoeficacia a favor de los hombres, y en sintomatología depresiva a favor de las mujeres. Esto es, los adolescentes varones presentaron un mayor puntaje en autoeficacia en comparación a las adolescentes mujeres, lo que ha sido verificado en otros estudios tanto internacionales (Choi, 2004; Reina, Oliva, \& Parra, 2010) como nacionales (Olivari \& Barra, 2005). Las mujeres presentaron una mayor sintomatología depresiva en comparación a los varones, lo que también concuerda con las investigaciones con muestras similares que han evidenciado que la sintomatología depresiva se presenta más frecuentemente en mujeres que en hombres, especialmente después de la pubertad (Barra, Cerna, Kramm, \& Véliz, 2006; Cova, Melipillán, Valdivia, Bravo, \& Valenzuela, 2007).

Una de las limitaciones de este estudio fue que la muestra fue no probabilística, y el rango de edad de la muestra de adolescentes, ya que solo abarcó el rango etario de 14 a 19 años de edad, es decir, no incluyó todo el intervalo de edad que define la etapa adolescente.

En resumen, se concluye que el BASH$B$, versión hispana, sería un instrumento que posee propiedades psicométricas adecuadas para medir barreras para la búsqueda de ayuda en población adolescente en nuestro medio sociocultural. Su utilización será de interés para la investigación del comportamiento de búsqueda de ayuda en población adolescente. En próximos estudios, se sugiere estudiar la estructura factorial 
identificada en otras muestras similares, y con adolescentes en un mayor amplio rango de edad.

Para finalizar, cabe destacar la relevancia que tiene el estudio de las barreras para la búsqueda de ayuda en población adolescente, ya que el tener mayor conocimiento de esta puede contribuir a diseñar intervenciones para que los jóvenes logren cada vez percibir menos barreras y buscar ayuda de manera oportuna.

\section{Referencias}

Abram, K., Paskar, L. D., Washburn, J. J., \& Teplin, L. A. (2008). Perceived barriers to mental health services among youths in detention. Journal of the American Academy of Child $\mathcal{E}$ Adolescent Psychiatry, 47(3), 301-308. https://doi.org/10.1097/CHI.0b01 $3 \mathrm{e} 318160 \mathrm{~b} 3 \mathrm{bb}$

Baker, G. (2007). Adolescents, social support and help-seeking behavior: An International literature review and program consultation with recommendations for actions. Ginebra: World Health Organization. Recuperado de http://apps.who.int/iris/bitstream/10665 /43778/1/9789241595711_eng.pdf

Barker, G., Olukoya, A., \& Aggleton, P. (2005). Young people, social support and helpseeking. International journal of adolescent medicine and health, 17(4), 315-335. https:/ /doi.org/10.1515/IJAMH.2005.17.4.315

Barra, E., Cerna, R., Kramm, D., \& Véliz, V. (2006). Problemas de salud, estrés, afrontamiento, depresión y apoyo social en adolescentes. Terapia psicológica, 24(1), 55-61.

Bentler, P. M. (1990). Comparative fit indexes in structural models. Psychological bulletin, 107(2), 238-246. http://doi.org/10.1037/00 33-2909.107.2.238

Boyd, C., Francis, K., Aisbett, D., Newnham, K., Sewell, J., Dawes, G., \& Nurse, S. (2007). Australian rural adolescents' experiences of accessing psychological help for a mental health problem. Australian Journal of Rural Health, 15(3), 196-200. https://doi.org/10.1 186/1471-244X-10-113
Brown, T. (2016). Confirmatory factor analysis for applied research (2da Edición). Guilford Press: Nueva York. Recuperado de http://p sycnet.apa.org/record/2006-07729-000

Burns, J. R., \& Rapee, R. M. (2006). Adolescent mental health literacy: young people's knowledge of depression and help seeking. Journal of adolescence, 29(2), 225-239. https://doi.org/10.1016/j.adolesce nce.2005.05.004

Carlisle, J., Shickle, D., Cork, M., \& McDonagh, A. (2006). Concerns over confidentiality may deter adolescents from consulting their doctors. A qualitative exploration. Journal of Medical Ethics, 32(3), 133-137. http://do i.org/10.1136/jme.2004.011262

Carlton, P. A., \& Deane, F. P. (2000). Impact of attitudes and suicidal ideation on adolescents' intentions to seek professional psychological help. Journal of Adolescence, 23(1), 35-45. https://doi.org/10.1006/jado. 1999.0299

Cartmill, T., Deane, F., \& Wilson, C. (2009). Gatekeeper training for youth workers: Impact on their helpseeking and referral skills. Youth Studies Australia, 28(1), 5-12. Recuperado de http://ro.uow.edu.au/cgi/viewcontent.cg i article $=1239 \&$ context $=$ hbspapers

Ciarrochi, J., Deane, F. P., Wilson, C. J., \& Rickwood, D. (2002). Adolescents who need help the most are the least likely to seek it: The relationship between low emotional competence and low intention to seek help. British Journal of Guidance and Counselling, 30(2), 173-188. https://doi.org /10.1080/03069880220128047

Cid, P., Orellana, A., \& Barriga, O. (2010). Validación de la escala de autoeficacia general en Chile. Revista médica de Chile, 138(5), 551-557. http://dx.doi.org/10.4067 /S0034-98872010000500004

Choi, N. (2004). Sex role group differences in specific, academic and general selfefficacy. The Journal of Psychology, 138(2), 149-159. https://doi.org/10.3200/JRLP.138 $.2 .149-159$ 
Cova, F., Melipillán, R., Valdivia, M., Bravo, E., \& Valenzuela, B. (2007). Sintomatología depresiva y ansiosa en estudiantes de enseñanza media. Revista Chilena de Pediatría, 78(2), 151-159. http://doi.org/10. 3200/JRLP.138.2.149-159

Eisenberg, D., Downs, M. F., Golberstein, E., \& Zivin, K. (2009). Stigma and help seeking for mental health among college students. Medical Care Research and Review, 66(5), 522-541. http://doi.org/10.1177/10775587 09335173

Gempp, R., Avendaño, C., \& Muñoz, C. (2004). Normas y punto de corte para la escala de depresión del centro para estudios epidemiológicos (CES-D) en población juvenil chilena. Terapia Psicológica, 22(2), 146-156. Recuperado de http://www.redaly c.org/pdf/785/78522205.pdf

Gulliver, A., Griffiths, K. M., \& Christensen, H. (2010). Perceived barriers and facilitators to mental health help-seeking in young people: a systematic review. BMC psychiatry, 10(113). https://doi.org/10.1186 /1471-244X-10-113

Horn, J. L. (1965). On the use of random variables in factor analysis. British Journal of Mathematical and Statistical Psychology, 19(1), 127-129. https://doi.org/10.1111/j.2 044-8317.1966.tb00361.x

Hunt, J., \& Eisenberg, D. (2010). Mental health problems and help-seeking behavior among college students. Journal of Adolescent Health, 46(1), 3-10. https://doi.org/10.1016 /j.jadohealth.2009.08.008

Kuhl, J., Jarkon-Horlick, L., \& Morrissey, R. F. (1997). Measuring barriers to help-seeking behavior in adolescents. Journal of youth and adolescence, 26(6), 637-650. https://doi.org/ 10.1023/A:1022367807715

Leshem, B., Haj-Yahia, M., \& Guterman, N. B. (2015). The characteristics of help seeking among Palestinian adolescents following exposure to community violence. Children and youth services review, 49, 1-10. https://d oi.org/10.1016/j.childyouth.2014.12.022

Lindberg, C., Lewis-Spruill, C., \& Crownover, R. (2006). Barriers to sexual and reproductive health care: urban male adolescents speak out. Issues in comprehensive pediatric nursing, 29(2), 73-88. http://doi.org/10.1080/01460 860600677577

Lindsey, M. A., Korr, W. S., Broitman, M., Bone, L., Green, A., \& Leaf, P. J. (2006). Helpseeking behaviors and depression among African American adolescent boys. Social Work, 51 (1), 49-58. https://doi.org/10.1093 /sw/51.1.49

Lorenzo-Seva, U., \& Ferrando, P. J. (2006). FACTOR: A computer program to fit the exploratory factor analysis model. Behavioral Research Methods, Instruments and Computers, 38(1), 88-91. https://doi.or g/10.3758/bf03192753

Lubman, D. I., Berridge, B. J., Blee, F., Jorm, A. F., Wilson, C. J., Allen, N. B . . . \& Wolfe, R. (2016). A school-based health promotion programme to increase helpseeking for substance use and mental health problems: study protocol for a randomised controlled trial. Trials, 17(393). https://doi. org/10.1186/s13063-016-1510-2

Luszczynska, A., Scholz, U., \& Schwarzer, R. (2005). The general self-efficacy scale: multicultural validation studies. The Journal of psychology, 139(5), 439-457. http://doi.or g/10.3200/JRLP.139.5.439-457

Lyren, A., Kodish, E., Lazebnik, R., \& O'Riordan, M. A. (2006). Understanding confidentiality: perspectives of African American adolescents and their parents. Journal of adolescent health, 39(2), 261-265. https://doi.org/10.1016/j.jadoheal th.2005.12.002

Olivari, C., \& Barra Almagiá, E. (2005). Influencia de la autoeficacia y autoestima en la conducta de fumar en adolescentes. Terapia Psicológica, 23(2), 5-11. Recuperado de http://www.redalyc.org/html/785/78523 201/

Organización Mundial de la Salud. (2010). Mental Health Promotion in Young People an Investment for the Future. Copenhague: Oficina Regional de Europa OMS. Recuperado de https://consaludmental.org/publicacion 
es/Mentalhealthpromotionyoungpeople.pd $\mathrm{f}$

Ranahan, P. (2010). Mental health literacy: A conceptual framework for future inquiry into child and youth care professionals' practice with suicidal adolescents. Child $\mathbb{E}$ youth care forum, 39(1), 11-25. https://doi.o rg/10.1007/s10566-009-9087-5

Raviv, A., Raviv, A., Vago-Gefen, I., \& Fink, A. (2009). The personal service gap: Factors affecting adolescents' willingness to seek help. Journal of Adolescence, 32(3), 483-499. https://doi.org/10.1016/j.adolesce nce. 2008.07.004

Reina, M. C., Oliva, A., \& Parra, A. (2010). Percepciones de autoevaluación: Autoestima, autoeficacia y satisfacción vital en la adolescencia. Psychology, Society Eु Education, 2(1), 55-69. Recuperado de http://repositorio.ual.es/bitstream/handl e/10835/2913/Reina\%20et\%20Al.pdf?sequ ence $=1$

Rickwood, D., Deane, F. P., Wilson, C. J., \& Ciarrochi, J. (2005). Young people's help-seeking for mental health problems. Australian e-journal for the Advancement of Mental health, 4(3), 218-251. https://doi.or $\mathrm{g} / 10.5172 /$ jamh.4.3.218

Sanjuán Suárez, P., Pérez García, A. M., \& Bermúdez Moreno, J. (2000). Escala de autoeficacia general: datos psicométricos de la adaptación para población española. Psicothema, 12 (Su. 2), 509-513. Recuperado de https://www.unioviedo.es/r eunido/index.php/PST/article/view/7741

Tishby, O., Turel, M., Gumpel, O., \& Pinus, U. (2001). Help-seeking attitudes among Israeli adolescents. Adolescence, 36(142), 249-264. Recuperado de https://search.proquest.com/openview/3 97c148a93d253d0ce50fdce061c1ae3/1?pq - origsite $=$ gscholar\&cbl $=41539$

Vogel, D. L, Wester, S. R., \& Larson, L. M. (2007). Avoidance of counseling: Psychological factors that inhibit seeking help. Journal of Counseling $\mathbb{E}$ Development, 85(4), 410-422. Recuperado de http://onlinelibrary.wiley.com/doi/10.10 02/j.1556-6678.2007.tb00609.x/full

Wilson, C. J., \& Deane, F. P. (2010). Helpnegation and suicidal ideation: the role of depression, anxiety and hopelessness. Journal of Youth and Adolescence, 39(3), 291-305. https://doi.org/10.1007/s10964-0 09-9487-8

Wilson, C. J., \& Deane, F. P. (2012). Brief report: Need for autonomy and other perceived barriers relating to adolescents' intentions to seek professional mental health care. Journal of Adolescence, 35(1), 233-237. https://doi.org/10.1016/j.adolesce nce.2010.06.011

Wilson, C. J., Deane, F. P., Ciarrochi, J., \& Rickwood, D. (2005). Measuring help-seeking intentions: Properties of the general help-seeking questionnaire. Canadian Journal of Counselling, 39(1), 15-28. Recuperado de http://cjcp.journalhosting.ucalgary.ca/cj c/index.php/rcc/article/viewFile/265/588

Wilson, C. J., Rickwood, D. J., Bushnell, J. A., Caputi, P., \& Thomas, S. J. (2011). The effects of need for autonomy and preference for seeking help from informal sources on emerging adults' intentions to access mental health services for common mental disorders and suicidal thoughts. Advances in Mental Health, 10(1), 29-38. https://doi.org /10.5172/jamh.2011.10.1.29

Wilson, C. J., Deane, F. P., Marshall, K. L., \& Dalley, A. (2008). Reducing adolescents' perceived barriers to treatment and increasing help-seeking intentions: effects of classroom presentations by general practitioners. Journal of Youth and Adolescence, 37(10), 1257-1269. https://doi .org/10.1007/s10964-007-9225-z

Wilson, C. J., Rickwood, D., \& Deane, F. P. (2007). Depressive symptoms and helpseeking intentions in young people. Clinical Psychologist, 11(3), 98-107. http://doi.org/1 0.1080/13284200701870954 


\section{Notas}

* Artículo de investigación. Esta investigación ha sido financiada por Conicyt, a través del Fondo Nacional de desarrollo Científico y Tecnológico del gobierno de Chile, Proyecto Fondecyt $\mathrm{N}^{\circ} 11130473$, concedido a la primera autora. 